Meta

Journal des traducteurs

Translators' Journal

\title{
Le soudage à l'arc sous gaz de protection
}

\section{Geneviève Henry}

Volume 31, numéro 4, décembre 1986

URI : https://id.erudit.org/iderudit/003056ar

DOI : https://doi.org/10.7202/003056ar

Aller au sommaire du numéro

Éditeur(s)

Les Presses de l'Université de Montréal

ISSN

0026-0452 (imprimé)

1492-1421 (numérique)

Découvrir la revue

Citer cet article

Henry, G. (1986). Le soudage à l'arc sous gaz de protection. Meta, 31(4),

407-420. https://doi.org/10.7202/003056ar d'utilisation que vous pouvez consulter en ligne.

https://apropos.erudit.org/fr/usagers/politique-dutilisation/ 


\section{LE SOUDAGE À L'ARC SOUS GAZ DE PROTECTION}

L'unité terminologique "soudage à l'arc sous gaz de protection " apparaît de prime abord comme relativement hermétique. Nous en étudierons le sens en découpant l'unité en ses différents composants, soit " soudage " (welding), "soudage à l'arc " (arc welding ; electric-arc welding ${ }^{1}$ ) ou "soudage électrique à l'arc" ${ }^{2}$, et "soudage à l'arc sous gaz de protection" (gas-shielded arc welding) ou "soudage à l'arc sous protection gazeuse ${ }^{3}$, et en fournissant tour à tour des explications sur ces mots.

\section{LE SOUDAGE}

Le soudage est une opération qui vise à assurer la continuité de la matière entre deux ou plusieurs pièces métalliques, de plastique, de verre, etc., antérieurement distinctes. Contrairement à ce que laissent supposer certains auteurs, le soudage n'est donc pas uniquement limité au seul domaine de la métallurgie. En effet, beaucoup de matières thermoplastiques sont aussi propres au soudage ${ }^{4,5}$. Par ailleurs, il est bon d'établir dès maintenant la différence entre les termes "soudage " et "soudure". Le soudage est une opération, la soudure est le résultat de cette opération. L'étude des suffixes nominaux "age " et "ure " tend d'ailleurs à corroborer cette distinction ${ }^{6}$.

Il existe aujourd'hui une gamme étendue de moyens servant à réunir entre eux différents éléments d'un assemblage. Ces moyens sont appelés "procédés de soudage" (welding process). La plupart des auteurs, tant anglophones que francophones, classent les différents procédés en deux grandes catégories : les procédés de soudage par fusion (fusion-welding process, fusion welding process ${ }^{7}$ ) ou procédés par fusion et les procédés de soudage par pression (solid-state welding process) ou procédés par pression. En fait, ces termes décrivent la façon dont la continuité de la matière est obtenue entre les pièces à assembler. Dans le premier cas, la continuité est obtenue par fusion des bords sans intervention de pression sur les pièces. Dans le deuxième cas, les surfaces à assembler sont écrasées et maintenues sous l'effort suffisamment longtemps pour assurer la cohésion. Précisons qu'il ne faut pas confondre "soudage avec pression" et "soudage par pression ". Dans le soudage avec pression, il peut y avoir ou non fusion des bords à assembler. Dans le soudage par pression, les bords demeurent à l'état solide, d'où l'expression anglaise "solid-state welding" ou "SSW" 8 .

Quoique très largenemnt utilisée tant en anglais qu'en français, la classification des procédés de soudage en procédés par fusion et procédés par pression laisse toutefois à désirer, puisqu'un groupe important de procédés ne peut y trouver sa place, soit les procédés de brasage (no equivalent). Tout comme le soudage, le brasage (no equivalent) est une opération qui vise à assurer la continuité de la matière entre les bords à assembler. Contrairement au soudage cependant, la liaison n'est obtenue ni par fusion des 
bords ni par pression. La constitution du joint est assurée uniquement par l'intervention d'un métal d'apport ( filler metal) dont la température de fusion est inférieure à celle des pièces à réunir. Le métal d'apport à l'état liquide mouille (to wet) les bords à assembler et en se solidifiant, constitue un joint. Les métaux de base (base metal'), c'est-à-dire le métal des pièces à réunir, ne participent donc jamais par fusion à la constitution de ce joint (voir figure 1). Le brasage n'est donc ni un procédé par fusion ni un procédé par pression.

Figure 1

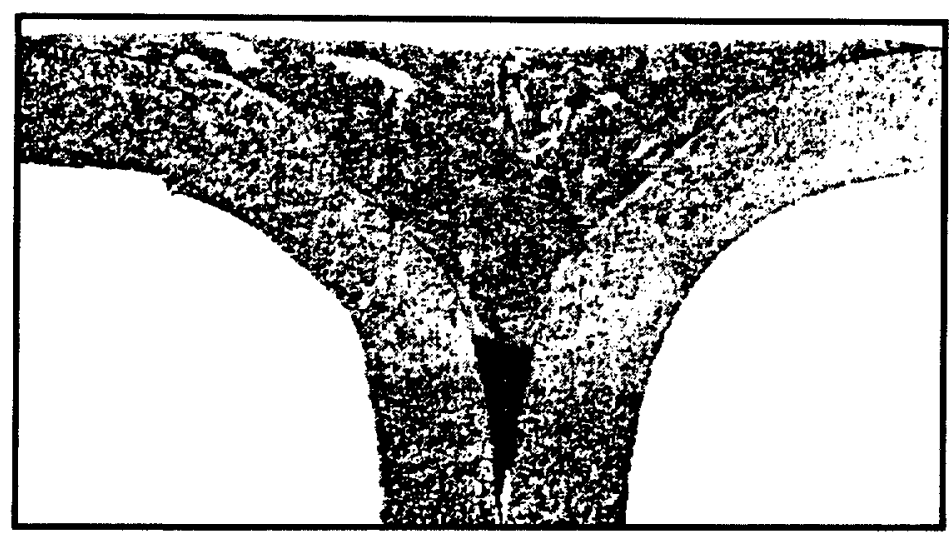

(TRIPR 75 9)

Le métal de base ne subit aucune modification au point du vue géométrique. Le joint est uniquement constitué grâce à l'intervention du métal d'apport qui s'accroche sur les bords à assembler.

C'est en établissant une distinction entre soudage autogène ou direct (no equivalent) et soudage hétérogène ou indirect (no equivalent) que l'on arrive à créer une classification plus adéquate. Selon Granjon, " est autogène » toute soudure qui a été exécutée selon un processus qui a provoqué une modification, ou la disparition du profil initial des pièces à assembler (par fusion, pression ou friction). Ainsi, au moins théoriquement, le terme autogène ne s'applique-t-il pas exclusivement aux gaz (comme c'est le cas dans d'autres pays et dans d'autres langues) ${ }^{10}$. Dans les procédés de soudage par fusion et les procédés de soudage par pression, il y a modification ou disparition du profil initial des pièces à assembler. On peut donc dire que le soudage par fusion et le soudage par pression sont en fait deux types de soudage autogène ou de soudage direct, la modification du profil des pièces à assembler étant le résultat d'une fusion (premier type), la disparition du profil des pièces étant le résultat d'une pression (second type). En d'autres termes, "soudage autogène " est un générique par rapport à "soudage par fusion " et à "soudage par pression", qui sont des spécifiques. De la même façon, on peut dire que les procédés de soudage par fusion et les procédés de soudage par pression sont des procédés de soudage autogène ou direct. Comme nous l'avons déjà vu, le brasage ne provoque aucune modification ni disparition du profil initial des pièces à assembler. De plus, le métal d'apport utilisé pour réaliser le joint ne possède pas la même composition chimique que le métal de base. Le brasage est donc du soudage indirect ou hétérogène ${ }^{11}$. Dans cette catégorie, on trouve le brasage fort (brazing; hard soldering12) où le métal d'apport possède une température de fusion inférieure à celle des pièces à réunir mais supérieure à $450^{\circ} \mathrm{C}^{13}$, le brasage tendre (soldering ; soft soldering ${ }^{14}$ ), où le métal d'apport possède une température inférieure à $450^{\circ} \mathrm{C}^{15}$, et le soudo-brasage (braze welding) 
ou soudobrasage qui s'apparente par la technique utilisée au soudage autogène par fusion et dont la température de fusion du métal d'apport est supérieure à $450^{\circ} \mathrm{C}^{16}$. Le tableau qui suit tente de résumer la situation. Il est à noter que les génériques " soudage autogène ; soudage direct ", "soudage hétérogène ; soudage indirect ; et brasage" ne possèdent pas d'équivalents en anglais ${ }^{17}$.

\section{FIGURE 2}

\section{PROCÉDÉS}

DE SOUDAGE

(WELDING PROCESS)

\author{
PROCÉDÉS DE \\ SOUDAGE \\ AUTOGENNE;
}

PROCÉDÉS DE
SOUDAGE
DIRECT
(NO
EQUIVALENT

PROCÉDÉS DE SOUDAGE HÉTÉROGÈNE PROCÉDÉS DE SOUDAGE

INDIRECT PROCÉDÉS DE BRASAGE (NO EQUIVALENT)

Tableau inspiré de COSOU 72176

\author{
PROCÉDÉS DE SOUDAGE PAR \\ FUSION ; \\ PROCÉDÉ PAR FUSION \\ (FUSION-WELDING PROCESS; \\ FUSION WELDING PROCESS)
}

PROCÉDÉS DE SOUDAGE PAR PRESSION ; PROCÉDÉ PAR PRESSION (SOLID-STATE WELDING PROCESS)

BRASAGE FORT

(BRAZING; HARD SOLDERING ;

B)

BRASAGE TENDRE

(SOLDERING ; SOFT SOLDERING ; S)

SOUDO-BRASAGE; SOUDOBRASAGE (BRAZE WELDING)

\section{LE SOUDAGE À L'ARC}

Pour obtenir la température ou la pression appropriées au soudage direct et indirect, plusieurs types d'énergies sont disponibles : chimique, mécanique, focalisée et électrique.

L'énergie chimique est celle que l'on obtient à la suite de la combustion d'un gaz, l'exemple le plus connu étant le soudage oxyacétylénique (oxyacetylene welding; $O A W^{18}$ ), ou à la suite de la réaction d'oxydation de l'aluminium, soudage aluminothermique (thermit welding). L'énergie mécanique est obtenue par explosion, friction, percussion, etc. (soudage par explosion (explosive welding), soudage par friction (friction welding), etc.). L'énergie focalisée est soit électrique, soudage par faisceau d'électrons (electron beam welding; electron-beam welding ${ }^{19}$ ), soit lumineuse, soudage laser (laser beam welding ; laser welding ${ }^{20}$ ). L'énergie électrique est celle qui, dans ce travail, nous intéresse en particulier. Elle est utilisée soit sous forme d'une résistance électrique, soit 
sous forme d'un arc électrique. Un arc électrique (electric arc; $\operatorname{arc}^{21}$ ) ou un arc est un courant intense qui s'établit entre deux surfaces conductrices, l'extrémité d'une électrode et la pièce à souder, par exemple (voir figure 3). Pour que l'arc puisse être amorcé (to initiate) et maintenu (to maintain) entre les pôles négatif et positif que constituent l'électrode et le point d'impact de l'arc sur la pièce, diverses conditions doivent être remplies, la plus importante étant que l'air ou les gaz entre l'électrode et la pièce soient ionisés, c'est-à-dire porteurs de charges électriques. L'arc électrique, ainsi nommé à cause de sa forme, donne lieu à toute une gamme de procédés de soudage à l'arc (arc-welding process; arc welding process ${ }^{22}$ ) ou procédé de soudage à l'arc électrique ${ }^{23}$. La relation qui existe entre "soudage » et "soudage à l'arc » est celle de générique à spécifique. En effet, le soudage à l'arc désigne un groupe particulier de procédés de soudage où la chaleur de fusion est fournie par un arc électrique jaillissant entre une électrode et la pièce à souder.

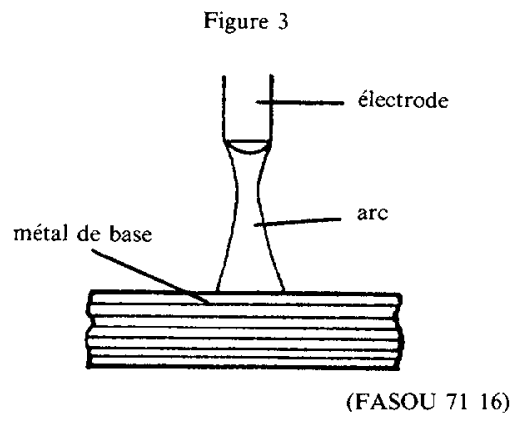

\section{LE SOUDAGE À L'ARC SOUS GAZ DE PROTECTION}

En présence de l'oxygène ou de l'azote contenus dans l'air, les métaux rouillent ou durcissent superficiellement. On parle alors d'oxydation et de nitruration. Lorsqu'on chauffe des pièces métalliques pour les souder entre elles, ces mêmes réactions chimiques se produisent. Elles ont un effet défavorable sur les caractéristiques du joint obtenu qui le rend généralement impropre à la constitution d'un assemblage. Pour éviter les défauts des soudures (welding defect), la plupart des procédés de soudage comportent un moyen de protection contre l'action nocive des gaz contenus dans l'air. En soudage oxyacétylénique par exemple, c'est la flamme qui, par son action réductrice, diminue d'au moins de moité le degré d'oxydation de la pièce à souder en rendant cette dernière moins électropositive, protège le bain de fusion (weld pool). En soudage à l'arc avec électrode enrobée (shielded-metal arc welding; stick-electrode welding ${ }^{24}$ ) ou soudage manuel à l'arc avec álectrode enrobée, c'est l'enrobage (coating) de l'électrode (produit chimique tel l'oxyde de titane, le carbonate de chaux, etc. $)^{25}$ qui, en fondant sous la chaleur intense dégagée, protège à la fois l'électrode, le métal en voie de transfert dans l'arc et le bain de fusion contre l'oxydation et la nitruration. Ces réactions peuvent aussi être évitées en soudant sous vide ou en utilisant une électrode fourrée, c'est-à-dire un feuillard enroulé sur lui-même et contenant un fiux solide dont le rôle est similaire à celui de l'enrobage des électrodes. En soudage à l'arc sous gaz de protection, c'est évidemment le gaz de protection (shielding gas; welding shielding gas ${ }^{26}$ ) qui sert à protéger le bain de fusion contre l'action de l'oxygène et l'azote de l'air. Le gaz de protection peut être soit chimiquement inerte, soit chimiquement actif. Les gaz inertes (inert gas) ne réagissent avec aucun autre corps. Les plus utilisés en soudage sont l'hélium (helium) et l'argon (argon). Les gaz actifs (active gas), comme leur nom l'indique, réagissent avec d'autres corps. Le dioxyde de carbone (carbon dioxide ; $\mathrm{CO}_{2}{ }^{27}$ ) ou di-oxyde de carbone ${ }^{28}$ est ce- 
lui qui est le plus utilisé. Pour pallier la présence de l'oxygène contenu dans ce gaz, les électrodes doivent comporter un enrobage encore plus chargé en éléments désoxydants. Des mélanges argon-hélium, dans le cas des gas inertes, et argon-oxygène ou argon-gaz carbonique, dans le cas des gaz actifs, sont également possibles. Le choix du gaz de protection est fonction d'un certain nombre de facteurs tels la nature du métal à assembler, son épaisseur, la forme du joint, la position de soudage, etc. Dans tous les cas, seuls des gaz possédant un très haut niveau de pureté, c'est-à-dire non contaminés par d'autres gaz ou par l'eau $\left(\mathrm{H}_{2} \mathrm{O}\right)$, sont utilisés, car les gaz de qualité ordinaire produiraient des défauts tels que la porosité dans les soudures. On dit par exemple de l'argon qualité soudage (welding-grade argon) ou de l'argon soudage, c'est-à-dire pur à $99,9 \%$, ou du $\mathbf{C O}_{2}$ qualité soudage (welding-grade $\mathrm{CO}_{2}$ ) ou du $\mathrm{CO}_{2}$ soudage, c'est-à-dire du gaz carbonique dont la teneur en azote est de l'ordre de $0,5 \%$.

\section{LE SOUDAGE À L'ARC SOUS GAZ DE PROTECTION}

L'unité terminoliguqe "soudage à l'arc sous gaz de protection " désigne un groupe de " procédés de soudage à l'arc dans lesquels l'arc et le bain de fusion sont isolés de l'atmosphère ambiante par une enveloppe de gaz provenant d'une souce extérieure "29 (voir figure 4). Il existe bien entendu des variantes de ce procédé de base. Celles-ci diffèrent principalement par la nature des électrodes utilisées, du gaz de protection, et du courant de soudage (continu ou alternatif). Les électrodes utilisées peuvent être consommables ou non (voir figure 5). Dans le premier cas, le métal de l'électrode est fondu et contribue pour une grande partie à la formation du joint. C'est le procédé GMAW (GMAW process ; gas-metal arc-welding process ${ }^{30}$ ) ou procédé de soudage GMAW. La nature du gaz de protection crée également de nouvelles variantes (voir figure 5). Ainsi, le procédé GMAW se subdivise de nouveau selon que le gaz utilisé est inerte ou actif. L'utilisation de gaz inerte donne lieu au procédé MIG (MIG process; M.I.G. process $^{31}$ ) ou procédé M.I.G. ${ }^{32}$ dont les initiales signifient Metal Inert Gas. L'utilisation de gaz actif donne

\section{FIGURE 4}

(welding)

$$
\text { soudage }
$$

brasage

(welding)

soudage direct; soudage autogène (no equivalent)

soudage par pression (solid-state welding ; SSW)

$$
\text { électrique chimique }
$$

soudage indirect; soudage hétérogène (no equivalent)

soudage par fusion (fusion welding) fort

brasage tendre

soudobrasage soudobrasage

résistance radiation conduction induction arc

s. à l'arc s. gaz de protection; s. à l'arc s. protection gazeuse ;

s. à l'arc s. gaz protecteur; $s$. à l'arc sous atmosphère gazeuse ;

s. à l'arc é s. atmos. gazeuse (gas-shielded arc welding) 
lieu au procédé MAG (no equivalent) dont les initiales signifient Metal Activ Gas. Dans le second cas, lorsque l'électrode est non consommable, cette dernière ne contribue en rien à la formation du joint. On utilise plutôt un métal d'apport, mais ce dernier n'est pas indispensable lorsque l'on soude des pièces peu épaisses. Il s'agit du procédé GTAW ( $G T A W$ process ; gas-tungsten-arc process ${ }^{33}$ ) ou procédé de soudage GTAW dont les initiales signifient Gas Tungsten Arc Welding. Lorsque le courant utilisé est contiunu, la polarité peut être soit directe (électrode négative) ou inverse (électrode positive). Il est également possible d'obtenir un courant pulsé. Lorsque le courant est alternatif, il peut être à fréquence industrielle $(60 \mathrm{~Hz})$, alternatif modulé, à moyenne fréquence ou à ondes rectangulaires.

\title{
FIGURE 5
}

\author{
soudage par fusion

électrique arc chimique \\ soudage à l'arc sous gaz de protection
}

VARIANTES

nature des électrodes

nature du gaz

protecteur nature du courant soudage GMAW ;

s. à l'arc s. protection gazeuse avec filélectrode fusible soudage GTAW ; sous protection gazeuse avec électrode réfractaire

soudage MIG ; soudage MAG, soudage TIG ; (v. synonymes dans lexique)

Le "soudage à l'arc sous gaz de protection " comprend également d'autres procédés. Ce sont le soudage au plasma (plasma arc welding ; plasma-arc welding ${ }^{34}$ ) ou soudage plasma ${ }^{35}$, le soudage à l'hydrogène atomique (atomic hydrogen welding; $A H W^{36}$ ) et le soudage électrogaz (electrogas welding ; $E G W^{37}$ ) ou soudage vertical en moule sous gaz de protection ${ }^{38}$.

Nous avons cherché à délimiter le champ sémantique du terme "soudage à l'arc sous gaz de protection ", qui est loin d'être clair. Dans un tableau résumant les procédés de soudage et les procédés connexes reproduit par $\mathrm{Smith}^{39}$, 1'American Welding Society inclut dans le terme "gas-shielded arc welding " les procédés GTAW, GMAW, PAW (plasma arc welding), EGW (electrogas welding), GCAW (gas carbon arc welding), SCAW (shielded carbon arc welding) et AHW (atomic hydrogen welding), mais se contredit dans sa définition normalisée du terme " $A$ general term used to describe gas metal arc welding, gas tungsten arc welding, and flux cored arc welding when gas shielding is used ${ }^{40}$. Plusieurs auteurs français, par ailleurs, semblent restreindre le champ sémantique du terme aux seuls procédés GTAW et GMAW41,42,43,44,45. (Ceci aurait pu être dû au fait que certains ouvrages (Mesou 1968, Encin 1973, Grans 1969) soient antérieurs à l'invention de certains procédés. Cette hypothèse ne peut s'avérer cependant, car la position des auteurs français est la même dans des ouvrages relativement récents (Gosou 1978, Gegen 1982).) La meilleure façon de savoir exactement ce que recouvre le terme "soudage à l'arc sous gaz de protection " est évidemment de consulter des définitions normalisées. Malheureusement, l'ACNOR, l'AFNOR et le Comité de normalisation de la soudure n'en ont rédigé aucune ${ }^{46,47}$. L'AFNOR a cependant édité une norme intitulée «Liste des procédés pour la représentation symbolique sur les dessins »48. D'après ce 
qui se dégage de cette dernière, sont compris dans le " soudage à l'arc sous gaz de protection " les procédés GMAW, FCAW, GTAW, AHW et PAW. Comme on peut le constater, les listes américaines et françaises ne concordent pas.

Le problème est donc de savoir si le terme recouvre uniquement les procédés faisant obligatoirement appel à un gaz de protection, comme le laissent sous-entendre les auteurs américains, ou pouvant exiger ou non un gaz de protection, comme le laissent supposer l'AFNOR en incluant dans sa liste le procédé FCAW, qui peut tantôt fonctionner avec un gaz de protection actif, tantôt sans gaz de protection. Les spécialistes québécois consultés ne s'entendent pas sur cette question, certains affirmant que seuls les procédés de soudage à l'arc nécessitant toujours un gaz de protection doivent être inclus dans le champ sémantique du terme, d'autres disant que tout procédé de soudage à l'arc fonctionnant à un moment donné avec du gaz de protection devait également en faire partie.

Faute de définitions et de positions précises sur ce terme, nous avons décidé, après mûre réflexion, de restreindre le champ sémantique de l'unité terminologique aux procédés de soudage à l'arc fonctionnant toujours sous gaz de protection pour les raisons suivantes. Premièrement, les procédés ne requérant pas obligatoirement un gaz de protection, comme le FCAW, ne pouvant d'après nous être logiquement dénommés " procédés de soudage à l'arc sous gaz de protection". De plus, si l'on s'en tient à la définition de l'American Welding Society qui inclut le FCAW dans le générique " gas-shielded arc welding» "when gas shielding is used» et à la norme éditée par l'AFNOR qui opine dans le même sens, il faudrait, en toute logique, inclure également tous les autres procédés qui peuvent également utiliser un gaz de protection mais de façon systématique. Inutile de dire que ces procédés sont nombreux et que le contenu sémantique du générique "soudage à l'arc sous gaz de protection " prendrait rapidement des proportions gigantesques. Notre position a par la suite été confirmée par deux unités de travail de l'Institut international de la soudure, soit la Commission VI et le Groupe d'étude "Information scientifique et technique "(GE-IST), pour lesquels le soudage à l'arc sous gaz de protection est " le terme générique recouvrant tous les procédés de soudage à l'arc dans lesquels on utilise toujours un gaz de protection $» \mathbf{4 9}$.

Nous examinerons maintenant brièvement les procédés de soudage compris dans le générique "soudage à l'arc sous gaz de proctection".

\section{LE SOUDAGE GMAW}

Dans le procédé de soudage à l'arc sous protection gazeuse avec fil-électrode fusible $^{50}$ (gas metal arc welding; gas-metal arc welding ${ }^{51}$ ), un arc jaillit entre l'extrémité d'un fil-électrode fusible ou consommable (qui se déroule automatiquement à travers une touche de soudage) et la pièce à souder. La protection du fil, de l'arc, du bain de fusion et des parties adjacentes du métal de base contre l'action nocive de l'atmosphère est assurée par l'écoulement d'un gaz provenant de la torche à travers la buse (voir figure 6). L'avantage principal de ce procédé est que le soudeur n'a pas à s'arrêter pour changer d'électrodes. De plus, c'est un procédé qui peut être facilement automatisé et même robotisé.

Le soudage à l'arc sous protection gazeuse avec fil-électrode fusible est un procédé dit semi-automatique (semiautomatic welding process) dans le sens où l'alimentation du fil-électrode se fait de façon automatique alors qu'un soudeur guide à la main la torche au-dessus du joint des pièces à assembler. Il se distingue ainsi des procédés manuels (manual welding process), dans lesquels le soudeur contrôle à la main tous les paramètres de soudage, et des procédés automatiques (automatic welding process), dans lesquels le soudeur n'a plus qu'un rôle de surveillant. Comme nous l'avons déjà vu, le soudage à l'arc sous protection gazeuse avec fil-électrode fusible comporte plusieurs variantes (fi- 


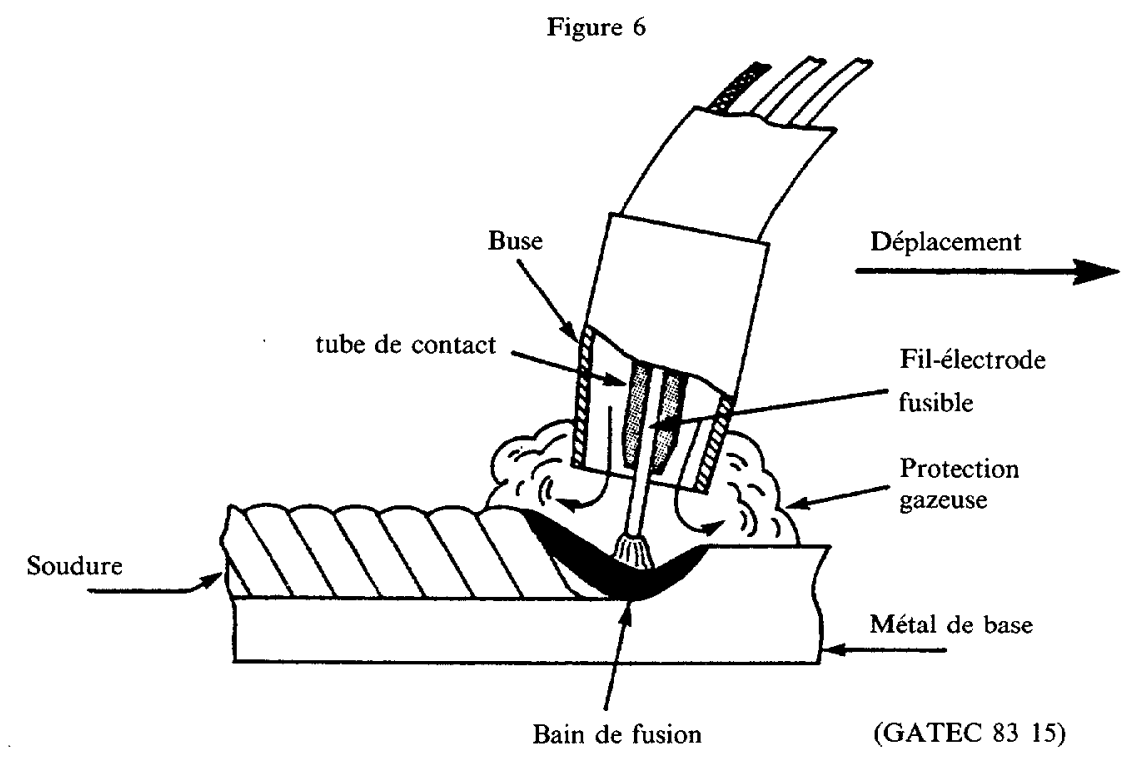

gure 5). Ces dernières peuvent être classées selon l'activité chimique du gaz protecteur [soudage MIG (MIG welding ; M.I.G. welding ${ }^{52}$ ) ou soudage M.I.G. ${ }^{53}$, soudage MAG (no equivalent)] ou selon le mode de transfert du métal dans l'arc. Ce dernier est une caractéristique fondamentale du procédé GMAW. Il est déterminé par le choix du gaz de protection, l'intensité du courant et la tension d'arc. Il existe trois modes de transfert du métal dans l'arc en soudage GMAW. Ce sont le transfert par court-circuit, le transfert globulaire et le transfert par pulvérisation axiale. Ces modes donnent respectivement lieu à des techniques que l'on peut distinguer grâce à l'adjonction de lettres minuscules au sigle GMAW, soit GMAW-c, GMAW-g, GMAW-pa. La description détaillée de ces variantes dépasserait, par sa complexité, le cadre de cet article. Aussi, nous contenterons-nous de ce bref aperçu du procédé de base. Aux États-Unis, le soudage à l'arc sous protection gazeuse avec fil-électrode fusible est désigné par le sigle GMAW, qui est également un générique. En Europe, on préfère utiliser les sigles MIG et MAG pour souligner la nature inerte ou active du gaz de protection utilisé. Comme le terme " gas metal arc welding " est un générique, nous croyons qu'il est préférable de le rendre également en français par un générique et d'utiliser les sigles MIG ou MAG uniquement lorsque l'on veut réellement parler des variantes et non pour désigner le procédé de base.

\section{LE SOUDAGE SOUS PROTECTION GAZEUSE AVEC ÉLECTRODE RÉFRACTAIRE}

Dans le soudage sous protection gazeuse avec électrode réfractaire (gas tungsten arc welding ; gas tungsten-arc welding ${ }^{54}$ ou soudage GTAW ${ }^{55}$, un arc est établi et maintenu entre une électrode de tungstène non consommable ou infusible et la pièce à souder (voir figure 7). Selon l'épaisseur du métal de base et la solidité du joint que l'on désire obtenir, on utilise ou non un métal d'apport. Ce dernier peut être soit une baguette tenue par le soudeur, soit un fil-électrode déroulé automatiquement à travers la torche lorsque le procédé est semi-automatique. Le gaz de protection joue le même rôle qu'en soudage GMAW. L'avantage de ce procédé est qu'il permet de souder sur de faibles épaisseurs un large éventail de métaux, y compris l'aluminium. On l'utilise lorsque l'on veut obtenir des soudures de haute qualité. 
Le soudage sous protection gazeuse avec électrode réfractaire comporte aussi plusieurs variantes, notamment le soudage TIG (TIG welding; T.I.G. welding ${ }^{56}$ ) ou soudage T.I.G. ${ }^{57}$, ce sigle étant formé des initiales de Tungsten Inert Gas (voir figure 5). En Europe, le soudage sous protection gazeuse avec électrode réfractaire est généralement appelé "soudage TIG". Dans les pays anglo-saxons, on le désigne par le sigle GTAW. Étant donné que le terme anglais est un générique, nous croyons qu'il est, encore une fois, préférable de le rendre en français par un générique.

Figure 7

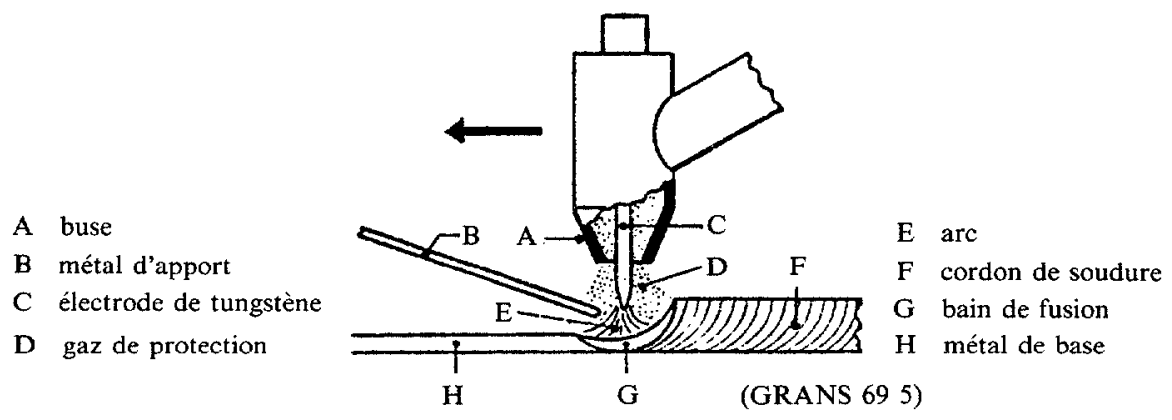

\section{LE SOUDAGE AU PLASMA}

Dans son sens général, le terme "plasma " désigne un gaz qui, après avoir été porté à des températures de l'ordre de plusieurs milliers de degrés, n'est plus composé de molécules ou d'atomes, mais d'ions positifs et d'électrons dans un état globalement neutre. Ainsi, l'arc de soudage (welding arc) ordinaire est un plasma à faible taux d'ionisation. Le soudage au plasma dérive du procédé GTAW (GTAW process ; gas-tugnstenarc process ${ }^{58}$ ) ou procédé de soudage GTAW. Contrairement à ce dernier cependant, l'arc qui est amorcé n'est pas un arc libre, c'est-à-dire ne rencontrant aucun obstacle entre l'extrémité de l'électrode et la pièce à souder, mais bien un arc confiné (constricted arc; constricted plasma arc ${ }^{59}$ ), c'est-à-dire un arc jaillissant entre une électrode de tungstène et une tuyère ou électrode creuse en cuivre de faible diamètre (voir figure 8 ).

En même temps, un gaz inerte, appelé gaz plasmagène, plasma gas ; plasmaforming gas ${ }^{60}$ ) ou gaz plasma, est projeté à très grande vitesse à travers cette tuyère. Cette dernière, tout comme l'électrode de tungstène, est refroidie par une circulation d'eau. Un second écoulement de gaz inerte assure la protection de l'électrode, de l'arc, $\mathrm{du}$ bain de fusion et des parties adjacentes du métal de base. Le confinement de l'arc dans un espace étroit accroît sensiblement sa température et, par conséquent, le taux d'ionisation du gaz plasmagène. On y observe des températures de l'ordre de 5000 à $18000^{\circ} \mathrm{C}$. Même si tout procédé de soudage à l'arc comporte nécessairement un plasma, ce terme réfère à l'" état d'énergie élevée d'un arc électrique éclatant dans un gaz dont le courant se trouve étranglé par passage à travers une section brusquement rétrécie $" 61$. Selon l'utilisation prévue du procédé (projection thermique, soudage ou coupage), la torche peut être conçue de façon que l'arc soit amorcé entre l'électrode de tungstène et la pièce - on parle alors d'arc transféré (transferred arc; transferred plasma $a r c^{62}$ ) - ou entre l'électrode de tungstène et la tuyère — on parle alors d'arc non transféré (non-transferred $a r c^{63}$ ) ou d'arc soufflé. 
Figure 8

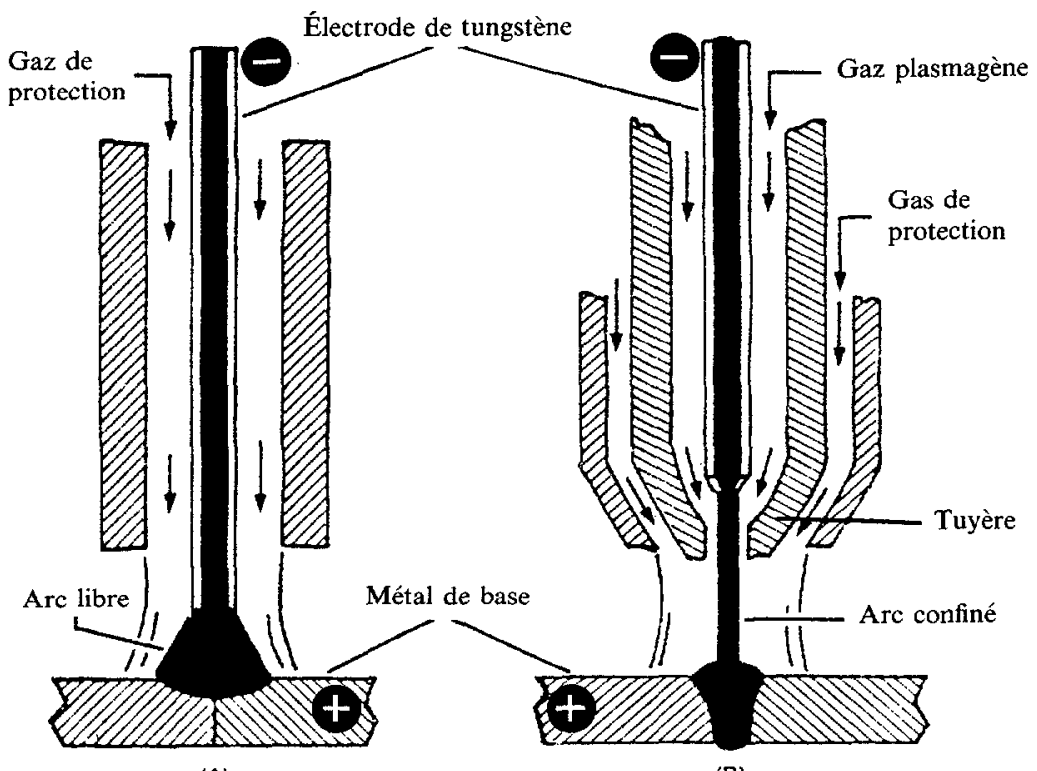

(A)

(B)

(SMIWE 84 348)

L'intérêt principal du procédé réside dans son effet de pénétration élevée donnant lieu à la technique du soudage en trou de serrure (keyhole welding). De plus, le soudage au plasma donne des soudures d'une qualité supérieure à celles que l'on obtient en soudage GTAW. On l'utilise surtout dans l'industrie aéronautique. Le coût extrêmement élevé de l'équipement constitue son principal désavantage.

\section{LE SOUDAGE À L'HYDROGÈNE ATOMIQUE}

Le soudage à l'hydrogène atomique (atomic hydrogen welding) est un procédé qui fonctionne également selon le principe de la dissociation des gaz à haute température. Aujourd'hui, il n'est presque plus utilisé pour des raisons d'ordre sécuritaire et économique.

Un arc électrique est établi entre deux électrodes de tungstène (voir figure 9). La protection des électrodes, de l'arc et du bain de soudure est assurée par deux écoulements d'hydrogène. Ce gaz joue à la fois le rôle de gaz protecteur et de gaz plasmagène. La chaleur de l'arc électrique a pour effet de dissocier les molécules d'hydrogène en ions positifs et négatifs, avec absorption de chaleur. Au contact de la pièce plus froide, la réaction inverse se produit avec dégagement de chaleur. Le résultat de ces deux réactions équivaut à un transport d'énergie beaucoup plus intense à partir des électrodes. La 
flamme produite en soudage à l'hydrogène atomique atteint ainsi une température de beaucoup supérieure à celle de l'arc ordinaire.

Figure 9

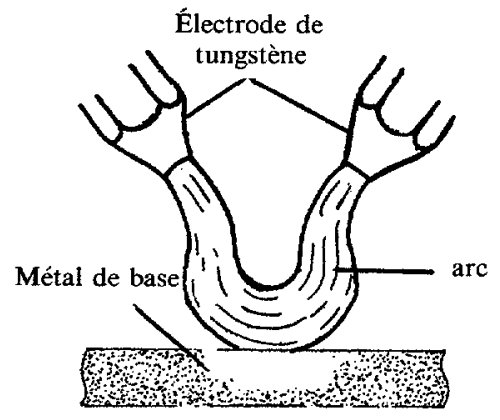

(MENAR 70 184)

\section{LE SOUDAGE ÉLECTROGAZ}

Le soudage électrogaz est un procédé entièrement automatique utilisé pour exécuter des soudures en position verticale sur tôles d'acier d'épaisseur moyenne (12 à 40 $\mathrm{mm}$ ). Pour ce faire, un fil-électrode consommable est déroulé verticalement à l'intérieur du joint. Les bords de ce dernier sont habituellement droits et écartés, mais ils peuvent également être chanfreinés. Entre l'extrémité du fil et le bain de fusion, un arc électrique jaillit sous protection gazeuse. Pour maintenir le métal fondu dans le joint, on utilise des lingotières (molding shoe) qui se déplacent vers le haut au fur et à mesure que le métal se solidifie. La nature du gaz de protection varie selon le métal soudé. S'il s'agit d'acier au carbone ou d'acier allié, on utilise du gaz actif ou un mélange de gaz actifs ; s'il s'agit d'acier inoxydable ou d'aluminium, on utilise un gaz inerte. Les fils-électrodes utilisés peuvent être pleins ou fourrés. Ce procédé a été conçu pour exécuter des soudures verticales ou légèrement inclinées, en particulier en construction navale (coques de navires), et en construction de réservoirs de stockage (grandes citernes, tours pour les industries chimiques et pétrolières), ainsi que dans d'autres applications moins courantes.

Figure 10

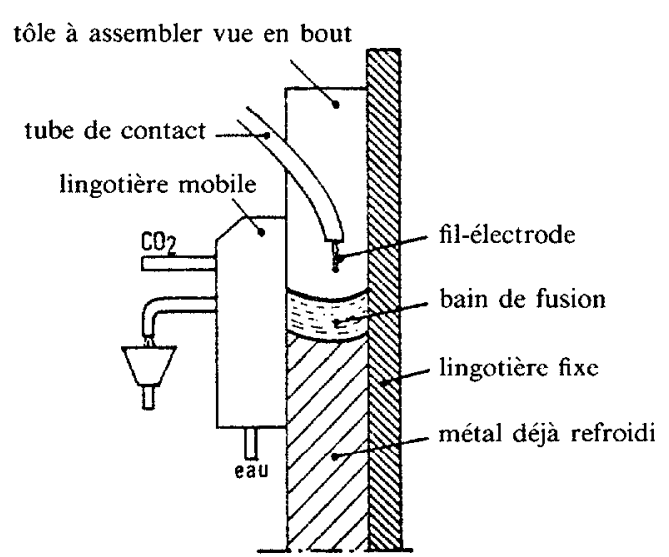

(FASON 71241 )
GENEVIÈVE HENRY Université de Montréal 
Notes linguistiques et techniques

1. SMIWE 84 172. Également: $A W S$; (AWS A3.0-80 2, SMIWE 84 19).

Les synonymes "arc-welding " (PEWEL 68 97), "electric-arc-welding" (PEWEL 68 97) et "electric arc welding " (SMIWE 84 99) sont à éviter car ils ne sont pas conformes à la règle d'utilisation des traits d'union dans les "adjective compounds" (voir FRAMO 72110 ). Nombreux sont les auteurs cependant qui font fi de cette règle tant dans leurs ouvrages que dans les normes techniques. Les langagiers sont alors aux prises avec des termes grammaticalement incorrects et pourtant normalisés.

2. Aussi : soudage à l'arc électrique.

3. GOSOU 786 ; soudage à l'arc sous gaz protecteur ; (GOSOU 7854 ) soudage à l'are sous atmosphère gazeuse ; (GRANS 69 5); soudage à l'arc en atmosphère gazeuse ; (GRANS 692); soudage à l'arc électrique sous atmosphère gazeuse (GRANS 69-5); soudage sous protection gazeuse (terme à éviter) ; (FASOU 71 13); soudage sous gaz (terme à éviter); (FASOU 71 197); soudage à l'arc sous flux gazeux (terme à éviter); (GOSOU 7854 ); soudage à l'arc en atmosphère contrôlée (terme à éviter); (GESOU 8024 ). Il existe bien d'autres procédés, tant électriques que chimiques, qui fonctionnent sous protection gazeuse. Il est donc faux de réserver le terme aux seuls procédés de soudage à l'arc.

La même remarque s'applique au second terme. De plus, ce dernier pourrait être confondu avec le terme "soudage aux gaz " qui désigne un groupe de procédés totalement différent.

Il ne faut pas confondre les termes " flux gazeux " et "protection gazeuse". Dans le premier cas, la protection est assurée par une matière chimique située à l'intérieur de l'électrode. Sous l'effet de la chaleur, cette matière se transforme en flux gazeux. Dans l'autre cas, la protection gazeuse provient toujours d'une source extérieure (bouteille de gaz). Le terme "soudage à l'arc en atmosphère contrôlée fait plutôt songer aux procédés de soudage à l'arc en enceinte fermée. Dans ces derniers, la protection gazeuse est réalisée de façon parfaite puisque le gaz est introduit à l'intérieur d'une enceinte étanche qui a préalablement été pompée à vide. Le soudeur opère de l'extérieur grâce à de longs gants souples."

4. COSOU 72172 .

5. GEGEN 824.

6. GREBO $6480,84$.

7. SMIWE 8420.

8. SMIWE 8419 , AWS A3.0-80 24.

9. Les pièces à assembler ne sont pas toujours des métaux. Lorsque tel est le cas, on utilise le terme «base material" en anglais (WHAND 76281 ). On pourrait le traduire par "matériau de base" en français.

10. GRANS 692.

11. COSOU 72174

12. BIMET 65 45, COSOU 71 176, (AWS A3.0-80 4, SMIWE 84 19).

13. ISO 857-1979 2.

14. BIMET 65 340, COSOU 72 176, (AWS A3.0-80 24, SMIWE 84 19).

15. ISO $857-19792$.

16. ISO $857-19792$.

17. COUSO 72175 .

18. SMIWE 84-19.

19. SMIWE $84635, E B W$, (SMIWE 84635 ).

20. SMIWE 84635 .

21. WEBDI 8071 .

22. GRASC 82 14-396, electric-arc welding process, (SMIWE 84-20, GRASC 82 14-596), electric arc-welding process, (SMIWE 84 290), electric-arc process (terme à éviter), (PEWEL 68 97). Ce terme ne précise pas s'il s'agit de soudage ou non. Il pourrait aussi bien décrire des procédés qui ne mettent pas en ceuvre le soudage, mais bien le brasage (ex. arc brazing, twin-carbon arc brazing).

23. procédé électrique à l'arc (terme à éviter). Même remarque qu'en 22.

24. SMIWE 84 364, SMAW, (SMIWE 84 23).

25. GESOU 8021.

26. SMIWE 8431 .

27. SMIWE 84448.

28. Aussi : $\mathrm{CO}_{2}$; gaz carbonique, anhydrique carbonique.

29. IISRE 86 ?

30. SMIWE 84 209, gas-metal-arc welding process, SMIWE 84 433), gas-shielded metal-arc welding process, (SMIWE 84 314).

31. PEWEL 68169.

32. Aussi : procédé SIGMA ${ }^{\text {nn }}$ (FRA); (MENAR 70 202); procédé ARGONAUT ${ }^{\text {nn }}$ (FRA); (MENAR $70202)$; procédé NERTALIC $^{\text {nn }}$ (FRA) ; (DUSOU 71219 ); procédé AIRCOMATIC ${ }^{\text {nn }}$ (FRA) ; (MENAR 70 202). 
33. SMIWE 84348.

34. SMIWE 84 347, GRASC 82 1-672, plasma welding, (SMIWE 84 255), PAW, (AWS A3.0-80 19, SMIWE 84 347).

35. Aussi : soudage au plasma d'arc; soudage à l'are plasma ; soudage au jet de plasma.

36. AWS A3.0-80 2, SMIWE 8423.

37. AWS A3.0-80 9, SMIWE 8423 .

38. Aussi : soudage vertical en moule sous gaz; soudage vertical sous gaz en caisson ; soudage vertical soutenu sous gaz.

39. SMIWE 8423.

40. AWS A3.0-80 12 .

41. GOSOU 7814.

42. MESOU 6811 .

43. ENCIN 73852

44. GEGEN 8210

45. GRANS 692.

46. Consultation téléphonique ACNOR (85-09-12).

47. Comité de normalisation de la soudure, communication personnelle (85-11-07).

48. AFPRO 78 .

49. Institut international de la soudure, communication personnelle (85-10-24).

50. Aussi : soudage à l'arc avec fil sous gaz de protection; soudage à l'arc avec fil plein sous gaz.

51. SMIWE 84420; gas metal-arc welding; (GRASC 82 14-597, GIWEL 73 70); GMAW; (AWS A3.0-80 12, SMIWE 84 420); gas-shielded metal-arc welding; (SMIWE 84 314); inert-gas-shieldedmetal-arc welding (terme à éviter); (GRASC 82 14-597). Le gas metal arc welding est un générique qui comprend des procédés qui ne fonctionnent pas uniquement avec des gaz inertes (inert gas) mais également avec des gaz actifs.

52. PEWEL 68169 ; MIG; (SMIWE 84 420); M.I.G.; (PEWEL 68 169); metal inert-gas arc welding; (SMIWE 84 430); metal-inert-gas arc welding; (SMIWE 84 56)

53. Aussi : soudage M.I.G.; soudage à l'arc sous protection de gaz inerte avec fil-électrode fusible ; soudage à l'arc avec fil-électrode en atmosphère inerte ; soudage sous gaz inerte avec fil-électrode consommable ; soudage avec fil-électrode en atmosphère inerte.

54. SMIWE 84 202, GRASC 82 14-598; gas-tungsten arc welding; (SMIWE 82 294); GTAW; (SMIWE 8423 ) ; GTA welding ; (SMIWE 84 202) ; gas-shielded tungsten-arc welding ; (SMIWE 84 313) ; inert gas tungsten arc welding; (AWS A3.0-80 14)

55. soudage à l'arc avec électrode réfractaire (terme à éviter). Il existe des procédés qui fonctionnent avec une électrode réfractaire sans gaz de protection (CAW).

56. PEWEL 68169 ; TIG; (SMIWE 84 313); T.I.G.; (PEWEL 68 169); tungsten-inert gas welding; (GRASC 82 671); tungsten-inert-gas welding; (SMIWE 84 313); tungsten-inert-gas arc welding; (SMIWE 84 56)

57. Aussi : soudage à l'arc en atmosphère inerte avec électrode de tungstène; soudage sous gaz inerte avec électrode de tungstène; soudage à l'arc électrique en atmosphère d'argon (FRA), soudage à l'argon (FRA), soudage à l'arc en atmosphère d'argon (FRA), soudage à l'arc en atmosphère inerte avec électrode à tungstène, soudage à l'électrode réfractaire (termes à éviter). Au Québec, les trois premiers synonymes ne devraient pas être utilisés car ils sont trop restrictifs. En effet, le gaz de protection utilisé n'est pas uniquement de l'argon. La construction syntaxique du quatrième synonyme est boiteuse. Même remarque qu'en 55.

58. SMIWE 84348.

59. SMIWE 84348.

60. SMIWE 84349 ; plasma-arc-forming gas; (SMIWE 84 349).

61. DUSOU 71232 .

62. SMIWE 84353.

63. SMIWE 84348.

Liste de codes de sources

AFPRO Association française de normalisation, Soudage et brasage des métaux. Liste des procédés pour la représentation symbolique sur les dessins, 1978.

AWS A3.0-80 American National Standard Institute/American Welding Society, Welding terms and Definitions, 1980.

BIMET Birchon, D. Dictionary of Metallurgy, 1965.

COSOU Couture, Bruno, "Quelques termes de soudage ", META, 1972. 
DUSOU Ducros, L., le Soudage manuel à l'arc électrique, 1971.

ENCIN Encyclopédie internationale des sciences et des techniques, 1973.

FASOU Syndicat national des fabricants de matériel de soudage, Guide du soudage, $t$. $V$, Soudage à l'arc sous gaz de protection (TIG, MIG, MAG), 1970.

FRAMO Frank, Marcella, Modern English. A Practical Reference Guide, 1972.

GEGEN Gerbeaux, H, Généralités sur le soudage, 1982.

GESOU Le Penven, Y. et H. Gerbeaux, Soudage. 1980,

GIWEL Giachino, J. W. et al., Welding Technology, 1973.

GOSOU Le Gouic, Roger, Précis de soudage, brasage et techniques connexes : coupage, métallisation, rechargement, 1978.

GRANS Granjon, H., "Soudage et soudabilité ", Techniques de l'ingénieur, 1969.

GRASC McGraw-Hill, Encyclopedia of Science and Technology, 1982.

GREBO Grevisse, le Bon Usage, 1964.

IISRE Institut international de la soudure. Commission VI "Terminologie ", Termes généraux. Recueil terminologique multilingue, 1986 (à paraître).

ISO 857-1979 International Standards Organization, Définition des procédés de soudage, 1979.

MENAR Mendel, L., Manuel pratique de soudage à l'arc, 1970.

MESOU Meslier, R., la Soudure autogène au chalumeau et à l'arc, 1968.

PEWEL Pender, James, Welding, 1968.

SMIWE Smith, Dave, Welding Skills and Technology., 1984.

TRIPR Triouleyre, J., Procédés de jonction.,1975.

WEBDI Webster's New World Dictionary, 1980.

WHAND Welding Handbook: Fundamentals of Welding I, 1976. 